\title{
Inclusión de condiciones socioculturales y factores de riesgo en la gestión de Key Energy Services ${ }^{*}$
}

\section{Cultural conditions and risk factors included in the management of Key Energiy Services}

Recibido: 22 de enero de 2014

Revisado: 22 de marzo de 2014 Aceptado: 27 de junio de 2014

Fabio Jose Ávila Dallos**

Nabors Drilling International

Sandra Milena Pinilla Bautista****

Universidad Pedagógica y Tecnológica de Colombia (UPTC)

\section{RESUMEN}

Key Energy Services es una compañía pionera a nivel mundial en los servicios de workover, completamiento y terminación de pozos petroleros, con 1200 taladros a nivel mundial y con el sueño de iniciar una línea de negocio sostenible y sustentable en Colombia.
Por ser una organización multinacional, Key Energy Services estandarizó su sistema de gestión bajo los requisitos establecidos en las normas técnicas ISO 9001, ISO 14001 y OHSAS 18001, con la expectativa de que todas sus operaciones se desarrollaran bajo un mismo y único lenguaje, de manera que las operaciones

\footnotetext{
* Artículo de resultado de investigación.

** Ingeniero Ambiental. Especialista en Higiene Industrial y Salud Ocupacional. Magíster en Calidad y Gestión Integral. Correo electrónico fabiodallos1@hotmail.com

****Ingeniera Industrial. Magíster en Calidad y Gestión Integral. Correo electrónico sa_mi_p@hotmail.com
} 
realizadas en el Medio Oriente se desarrollaran en forma similar en Colombia. La alta dirección asumió esta responsabilidad y, siguiendo la directriz impartida, logró la certificación de sus sistemas de gestión, sin embargo, los resultados no fueron los esperados. Entre los años 2011 y 2014 se perdieron dos contratos, hubo que despedir a más de 400 trabajadores, se presentó una caída de la acción en la Bolsa superior al $200 \%$ y los índices de accidentalidad laboral, ambiental y operacional se incrementaron, lo cual hizo perder la confianza de los clientes en Key Energy Services y la posibilidad para la empresa de abandonar sus operaciones en Colombia.

Como parte del análisis de la anterior situación se vio la necesidad de involucrar las condiciones socioculturales de las personas que forman parte de la organización, como entrada para la planificación del sistema de gestión integrado, con el fin de complementar algunos aspectos humanos que no son tradicionalmente incluidos dentro de los sistemas de gestión. Pese a los inconvenientes por la falta de información nacional e internacional sobre el tema, esta propuesta incluye y evalúa las condiciones y los factores de riesgo socioculturales en el sistema integrado de gestión de Key Energy Services, para responder luego algunos interrogantes, por ejemplo: ¿Por qué, a pesar de la voluntad de la alta dirección para que los sistemas de gestión se diseñen e implementen correctamente, los resultados no son los esperados? ¿Qué papel cumplen las condiciones socioculturales para el logro de esos resultados? Para Key Energy Services, conocer las condiciones socioculturales de los trabajadores — sin importar su cargo, su estrato social, sus creencias o asociaciones, entre otros- resultó importante como estrategia para adaptar y mejorar el sistema de gestión integrado, bajo la premisa de que son los trabajadores quienes garantizan el éxito o el fracaso de los sistemas de gestión en la organización.
Palabras clave: sistema integrado de gestión, riesgo sociocultural, HSEQ.

\section{ABSTRACT}

Key Energy Services is a worldwide pioneer in workover services, completion of oil wells, with 1200 rigs in the world and with the dream of starting a sustainable business line in Colombia.

As a multinational organization, Key Energy Services has standardized its management system under the requirements of the technical standards ISO 9001, ISO 14001 and OHSAS 18001, with the expectation that all operations are carried out under one and the same language, so that the operations in the Middle East be developed in a similar way in Colombia. Senior management assumed this responsibility and following the given guideline, achieved certification of their management systems, however the results were not as expected. Between 2011 and 2014 two contracts were lost, they had to lay off more than 400 workers, shares on the stock exchange fell $200 \%$ and the rates of labor, environmental and operational accidents were increased, which made lose the trust of customers in Key Energy Services and the possibility for the company to abandon its operations in Colombia. The analysis of the previous situation show the need to involve socio-cultural conditions of the people's organization, as input for planning integrated management system, in order to supplement some human aspects that are not traditionally included in management systems. Despite the drawbacks by the lack of national and international information on the subject, this proposal includes and assess conditions and risk factors sociocultural in the integrated management system of Key Energy Services, to answer some questions such as: 
Why which despite the willingness of senior management to designed and implemented management systems correctly, the results are not what you expected ?, What is the role of the sociocultural conditions for achieving those results ?. For Key Energy Services was important to know the sociocultural conditions of workers, regardless of their position, their social status, beliefs or associations, among others, as a strategy to adapt and improve the integrated management system under the premise that they are workers who guarantee the success or failure of management systems in the organization.

Keywords: Socio-cultural risk, integrated management system, HSEQ.

\section{INTRODUCCIÓN}

Una autocrítica a partir de los errores cometidos y de las lecciones aprendidas por la compañía se refiere a la necesidad de conocer cuáles son las áreas de influencia en las que se desarrollan los diferentes contratos, porque los comportamientos sociales y las condiciones culturales no son iguales en todas partes y porque el éxito de un sistema de gestión depende de sus trabajadores, cuyo comportamiento está influenciado por la cultura de cada país y de cada región.

Desarrollar el modelo de gestión integral involucrando las condiciones socioculturales de las regiones de Colombia permite mejorar los resultados de la organización Key Energy Services (KES) en aspectos de calidad, salud ocupacional, seguridad industrial y medio ambiente, optimizando y mejorando la calidad de vida de sus trabajadores y el desempeño de la organización en los aspectos mencionados, asegurando la sostenibilidad y sustentabilidad de la organización.
Condiciones socioculturales como los niveles de estudio, las asociaciones a grupos sindicales, la frecuencia de ausentismo, el estrato económico, las motivaciones de los trabajadores, entre otras, le permiten a la organización determinar acciones y controles necesarios para su desempeño. Por ejemplo, si se determinara que existe dentro de la población trabajadora un $80 \%$ de personal que es oriundo de la región, se haría necesario establecer una fuerte política laboral de contratación, la cual debería ser trasladada a los requisitos contractuales; si se conociera que la mayoría de sus trabajadores son personas mayores de 50 años, pero cumplen con el requisito social de pertenecer a la región, la organización podría mejorar sus profesiogramas y perfiles de cargo.

Estas acciones no deben considerarse como excluyentes al momento de realizar una contratación, simplemente se deben tener en cuenta para que la organización conozca y controle todas estas variables críticas que determinarán su sostenibilidad.

El estudio se realiza en diferentes etapas:

La primera de ellas inicia con la verificación de la presencia explícita de condiciones socioculturales en el sistema de gestión integrado (SGI) de Key Energy Services o en los requisitos contractuales. Se realiza una revisión documental y se determina que en ningún requisito se establece la necesidad de conocer las condiciones socioculturales de las regiones.

La segunda etapa se centra en la obtención de información por medio de una encuesta con la cual se pretende listar, mediante revisión documental teórica, las condiciones socioculturales de los trabajadores que deben ser incluidas en el SIG de Key Energy Services; entre ellas se encuentran el nivel de educación, la frecuencia de uso de la EPS por enfermedad común a laboral, el tipo de organización a la que pertenece la población trabajadora,el conocimiento del manual 
de funciones, la capacitación y/o formación, el conocimiento del SIG. El análisis de esta información se envía a la organización, bajo el esquema de lineamientos convenientes extraídos del análisis de la encuesta.

La tercera etapa del proyecto se centra en diseñar una matriz para la valoración de factores de riesgos socioculturales en la ejecución de proyectos de workover 0 perforación para la empresa KES, basada en la Guía para la identificación y valoración de peligros GTC 45 (ICONTEC, 2012) y validada en su contenido por un grupo de expertos técnicos conformado por un experto en el sector de hidrocarburos, un experto en sistemas de gestión y una experta en literatura y lenguaje.

Finalmente, en la última etapa del proyecto se elabora una propuesta metodológica para la inclusión de las condiciones socioculturales y la matriz de valoración de factores de riesgo en el SIG de Key Energy Services, según el ciclo de mejora continua y con validación de su contenido.

Articular los sistemas de gestión con las condiciones socioculturales permite garantizar la identificación de los requisitos de las partes interesadas para lograr crear políticas y objetivos estratégicos que establecen el norte y aseguran la sostenibilidad de una organización.

Se elabora y ejecuta una propuesta que contempla las condiciones socioculturales y la identificación y evaluación de riesgos en el SIGH de la población trabajadora de la compañía KES. Para hacer esto posible, se procedió a listar e incluir, mediante revisión documental teórica, estas condiciones en el SIG de la empresa. Posteriormente, por medio de la aplicación de una encuesta a la muestra de estudio, previa validación de contenido del instrumento, se diseña una matriz para la valoración e inclusión de factores de riesgo socioculturales en la ejecución de proyectos de workover o perforación para KES, basados en la guía GTC45. Seguidamente, se verifica la utilidad de este listado entregándole a la compañía los lineamientos que permiten evidenciar la presencia de las condiciones socioculturales en el SIG de KES. Con base en estos resultados, se elabora una propuesta metodológica que contempla las condiciones socioculturales de los trabajadores y la matriz de valoración de factores de riesgo según el ciclo de mejora continua, con validación de su contenido.

\section{METODOLOGÍA}

El presente trabajo corresponde a una investigación cualitativa. Como hipótesis se estableció que el conocimiento de los trabajadores de las comunidades en donde se desarrollan las operaciones de Key Energy Services se hace indispensable no solo para poder diseñar una matriz de inclusión y valoración de condiciones y factores de riesgos socioculturales, sino para que su implementación permita eliminar las actuales prácticas inconvenientes que se llevan a cabo en la compañía.

Las variables utilizadas en el desarrollo de este proyecto fueron enfocadas en condiciones socioculturales del área donde se desarrollan los proyectos, como género, edad, estrato socioeconómico, nivel de educación, incapacidades médicas de origen laboral, percepción del cumplimiento de normas y reglamento, lazos de confianza con los compañeros y líderes de la organización y de conocimiento, por ejemplo: conocimiento del manual de funciones, cumplimiento de lo estipulado en manuales, procedimientos normas establecidas por la organización, satisfacción en el cargo que ocupa actualmente, temas o intereses para capacitación, conocimiento del SIG, comprensión de la información suministrada por la empresa, aplicación del SIG con condiciones socioculturales, el trabajo basado en la experiencia supera el aprendizaje formal, beneficios de 
la implementación del SIG, implementación y mantenimiento exitoso del SIG en la organización, condiciones sociales que pueden ayudar en la implementación y mejora del SIG, contribución social de las empresas en la comunidad, contribución del SIG en el desarrollo de la comunidad, influencia del orden público en el entendimiento, aceptación e implementación del SIG.

Los indicadores estarín relacionados con las variables anteriormente mencionadas.

La población correspondió a trabajadores de la empresa Key Energy Services, y para la muestra se utilizó un muestreo aleatorio simple (MAS), que correspondió a 53 trabajadores del total de la población, de 400 trabajadores aproximadamente.

El trabajo utilizó dos revisiones documentales: una para elaborar el marco teórico y otra sobre documentos de Key Energy Services, con el fin de verificar si en el SIG de la organización se encontraban entradas para condiciones socioculturales de los trabajadores; una encuesta con preguntas de la lista de condiciones aplicada a la muestra para elaborar, con los resultados, unos lineamientos útiles para la compañía, la Guía técnica colombiana 45 /2012 y el Ciclo de Deming.

De acuerdo con Loreto Marchan y Aldo del Río (2014), la diversidad cultural en las organizaciones viene concebida así: "la diversidad en la empresa es un reflejo de variables demográficas, de comportamiento cultural, actitudes, normas y valores existentes en una sociedad. La diversidad se producirá en la empresa cuando las características socioculturales que definen a la sociedad en la que dicha organización actúa se reflejan en la plantilla de trabajadores".

Otros investigadores, como Williams y O'Reilly, afirman que, si por el contrario, las organizaciones no tienen en cuenta los diferentes aspectos concernientes a la diversidad, podrían generar efectos nocivos en la comunicación, en el manejo de conflictos, en la rotación, el ausentismo y en la toma de decisiones.

Estos pensamientos soportaron la hipótesis del proyecto, según la cual "la inclusión de las condiciones socioculturales de los trabajadores en el Sistema Integrado de Gestión de Key Energy Services elimina prácticas inconvenientes que se llevan a cabo en la compañía", condiciones reflejadas en los resultados de alta accidentalidad, tiempo productivo perdido, disminución de credibilidad con el cliente, entre otros.

\section{RESULTADOS Y DISCUSIÓN}

El desarrollo de este proyecto permitió elaborar una propuesta metodológica para la inclusión y valoración de condiciones y factores de riesgos socioculturales en el sistema integrado de gestión (SIG) de Key Energy Services.

Para desarrollar esta propuesta, se partió de una hipótesis confirmada, relacionada con la ausencia de las condiciones socioculturales en el sistema de gestión integral de la organización, donde se revisaron los criterios del cliente y la documentación del sistema de gestión de la organización y se evidenció la ausencia total de las condiciones socioculturales. Posteriormente se plantearon algunos elementos de entrada para la inclusión de condiciones socioculturales en el SIG de Key Energy Services, esto como resultado de la encuesta aplicada a las regiones de Barrancabermeja y Puerto Boyacá.

Estos elementos, validados por un grupo de expertos (en sistemas de gestión, en estadística y en trabajo social), permitieron diseñar la matriz para la valoración de factores de riesgos socioculturales en la ejecución de proyectos de workover. 
De acuerdo con el análisis desarrollado con la encuesta como instrumento de recolección de información, se sugieren los siguientes lineamientos relevantes:

- Nivel de educación: Predominan los niveles educativos de bachillerato y de carreras técnicas. Las condiciones del trabajo por rotación de turnos se convierten en una razón que justifica la dificultad para que el personal mejore sus competencias basadas en la academia. Esta situación justificada dificulta la interpretación y asimilación de los sistemas integrados de gestión, ya que estos no son diseñados teniendo en cuenta la educación formal de sus trabajadores. Con esta variable se podrían modificar la matriz de entrenamiento, el establecimiento de competencias de personal de acuerdo con el nivel de estudio y las estrategias de implementación del SIG.

- Frecuencia de uso de la EPS por enfermedad común a laboral: Esta variable muestra una parte del comportamiento social y las condiciones físicas de los trabajadores quienes, por su edad, tienen con mayor frecuencia incapacidades médicas. Este indicador podría ser una entrada para la modificación de profesiogramas y perfiles de cargo de la organización.

- Tipo de organización a la que pertenece la población trabajadora: Las relaciones sociales de los trabajadores predominan en grupos culturales, sociales y sindicales. Aunque no todo el personal pertenece al grupo sindical llamado USO, las decisiones que tome la Unión Sindical Obrera si afectan a todos los trabajadores del área, tanto en su comportamiento como en su desarrollo laboral. Aunque existe el derecho de los trabajadores a estar afiliados a un grupo sindical, esta variable puede ser una entrada para modificar las políticas laborales y de contratación de la compañía sin ser excluyente por su orientación social. Adicionalmente, esta variable le muestra a la organización una fortaleza para el establecimiento de políticas, procesos y programas estratégicos, para garantizar que con su implementación se alcancen los objetivos esperados.

- Grado de confianza con líderes y compañeros de trabajo: Esta variable debería mostrar un $100 \%$ de grado de confianza con líderes y equipo de trabajo. Esta variable indica que el proceso de comunicación, la estructura y la cultura organizacional deben ser replanteadas para garantizar un ambiente laboral seguro y sostenible en la organización.

- Conocimiento del manual de funciones: Esta variable debería mostrar un $100 \%$ de entendimiento de funciones y claridad organización. Esta variable obliga a la organización a establecer estrategias de entrenamiento y mejora de sus procesos de selección, evaluación y contratación de personal, así como la inclusión de temas específicos de acuerdo con los perfiles de cargo de cada trabajador, gestionados por medio de su matriz de capacitación.

- Capacitación y/o formación: El $100 \%$ de la población trabajadora manifiesta su interés en capacitarse y mejorar sus competencias laborales, sin embargo, el interés se refiere a conocimientos técnicos operativos, y un porcentaje mínimo manifiesta tener un interés ambiental y en HSE.

Con esta variable se pueden replantear la identidad y los valores de la organización con sus respectivas estrategias de comunicación. Para la organización, la seguridad de las personas y el medio ambiente es el pilar número uno, sin embargo, los trabajadores muestran un interés generalizado en la operación; aunque no se podría afirmar que esta tendencia sea buena o mala, síse invita a la organización a generar estrategias para resaltar la importancia de los temas en QHSE, ya que allí se origina el cambio hacia los 
sistemas de gestión integrales. Adicionalmente, le muestra a la organización la aspiración del personal en procesos de ascenso, con lo cual la organización puede mejorar su proceso de selección, evaluación y contratación de personal.

- Conocimiento del SIG: Para más del $50 \%$ de la población, el significado y los enfoques de un sistema de gestión integrado no son claros por los miembros de la organización. Con esta variable, la organización puede mejorar su proceso de selección, evaluación y contratación de personal, así como mejorar su matriz de capacitación y entrenamiento por cargo, al adecuar sus perfiles de cargo a la oferta y a la demanda laboral que se tienen en las áreas de influencia de los proyectos.

- Planificación del SIG con personal involucrado: La población trabajadora manifiesta su interés en contribuir en la planificación del SIG. Esta variable le permite a la organización diseñar un sistema de gestión integral con todas las partes involucradas e interesadas.

\section{CONCLUSIONES}

De acuerdo con la revisión documental, se evidenció que el SIG de la empresa Key Energy Services no incluye las condiciones socioculturales de los trabajadores. En consecuencia, s necesario identificar las condiciones socioculturales y valorar los riesgos socioculturales en el diseño y la implementación del sistema integrado de gestión, con el fin de entender y conocer esas condiciones de los trabajadores. Se encontró que los trabajadores, por múltiples condiciones, no siempre comprenden las razones por las cuales se debe proceder de una determinada manera en el SIG, lo que hace lentos los procesos, genera no conformidades y causa dificultades en la administración de la organización.

Los elementos de entrada para la inclusión de las condiciones socioculturales en el SIG de Key Energy Services son una propuesta para que la organización los acoja 0 incluya con el fin de planificar y controlar los elementos que considere determinantes para la ejecución de sus actividades.

Como las normas técnicas internacionales ISO 9001, ISO 14001 y OSHAS 18001 no determinan claramente la importancia de conocer las condiciones socioculturales de los trabajadores y en ciertas circunstancias, como la perforación de pozos petroleros en zonas aisladas, este es un factor decisivo para la operación satisfactoria del SIG. Esta propuesta contribuye a la identificación y valoración de esas condiciones socioculturales como un aporte metodológico en la planificación del SIG.

La hipótesis planteada en el trabajo de investigación continúa en desarrollo, ya que esta depende de la implementación de la metodología para la valoración de los factores de riesgo socioculturales en el SIG de la empresa Key Energy Services y de los resultados que se obtengan al realizarla.

\section{REFERENCIAS}

Abril, C., Palomino, A. y Sánchez, J. (2006). Manual para la integración de sistemas de gestion: Calidad, medio ambiente y prevención de riesgos laborales. España: Editorial Fundación Confemetal.

Ayala, J. (2003). Información de sociedad: Del suceso a la calidad de vida. Cataluña: Servei de Publicacions. 
Atehortúa, F., Bustamante, R. y Valencia, J. (2009). Sistema de gestión integral. Una sola gestión, un solo equipo. Medellín: Universidad de Antioquia.

Bourdieu, P. (2001). Poder, derecho y clases sociales (2.a ed.). España: Editorial Desclée de Brower S. A.

Cecchini, S. (2005). Indicadores sociales en América Latina y el Caribe. Santiago de Chile: CEPAL.Recuperado de http://www.cepal.org/publicaciones/ xml/0/23000/lc12383e.pdf

Cegarra, J. (2011). Metodología de la investigación cientifica y tecnológica. Madrid: Ediciones Díaz de Santos S. A.

Congreso de la República de Colombia. (1979). Ley 9 de 1979. Por la cual se dictan Medidas Sanitarias. Recuperado de goo.gl/pzCSE

Cortina, A. (2011). Alianæa y contrato: Política, ética y religión (2.a ed.). Madrid: Trotta.

Daft, R. (2007). Teoría y diseño organizacional. (9.a ed.) México: Cengage Learning Editores S. A.

Grande, I. y Abascal, E. (2009). Fundamentos y técnicas de investigación comercial (10.a ed.). Madrid: Esic Editorial.

Gallino, L. (2005). Diccionario de sociología (3.a ed.). México: Editores Siglo XXI.

Gonzáles, R., Barron, M. (2007). Experiencias de desarrollo rural. Dos visiones de vinculación universitaria: Colima y Iowa. México: Editorial de la Red Nacional de Investigación Urbana.

Hernández, R., Collado, C. y Baptista, P. (2007). Metodología de la investigación (4.a ed.). México: McGraw Hill Interamericana.
Herrero, J. (2014). ¿Qué es cultura? Recuperado de http://pnglanguages.org/training/capacitar/antro/ cultura.pdf

Instituto Colombiano de Normas Técnicas y Certificación - ICONTEC. (2005). Sistema de gestión de la calidad. Fundamentos y vocabulario. Bogotá: ICONTEC.

(2004). Norma técnica colombiana NTC ISO 14001. Sistemas de gestión ambiental: requisitos con orientación para su uso. Bogotá: ICONTEC.

(2007). Norma técnica colombiana OHSAS 18001. Sistemas de gestión en seguridad y salud Ocupacional: requisitos. Bogotá: ICONTEC.

Lillo, N. y Roselló, E. (2001). Manual para el trabajo social comunitario. España: Editorial Narcea.

Marchant, L. y Del Río, A. (2014). Gestión estratégica de la diversidad cultural en las organizaciones. Ciencias Sociales On Line. Revista Electrónica. Recuperado de http://www.uvm.cl/csonline/2008_1/ marchant.pdf

Ministerio de Gobierno de la República. (1994). Decreto 1295 de 1994. Por el cual se determina la organización y administración del Sistema General de Riesgos Profesionales. Diario Oficial No. 41.405 de junio 24 de 1994. Recuperado de goo. $\mathrm{gl} / \mathrm{bV} 531 \mathrm{~N}$

Ministerio de la Protección Social. (2007). Resolución 1401 de 2007. Por la cual se reglamenta la investigación de incidentes y accidentes de trabajo. Diario Oficial 46638 de mayo 24 de 2007. Recuperado de g0o.gl/ewN96K 
Ministerio de Salud. (1992). Resolución 4225 de 1992. Por la cual se adoptan unas medidas de carácter sanitario al Tabaquismo. Recuperado de goo. $\mathrm{gl} / 1 \mathrm{QcxNR}$

Ministerio de Trabajo. (1967). Decreto 13 de 1967. Por el cual se incorporan al Código Sustantivo del Trabajo las disposiciones de la Ley 73 de 1966. Diario Oficial 32.131 de 25 de enero de 1967. Recuperado de goo.gl/eWsoM2

Ministerio de Trabajo y Seguridad Social. (1979). Resolución 2400 de 1979. Por la cual se establecen algunas disposiciones sobre vivienda, higiene y seguridad en los establecimientos de trabajo. Recuperado de goo.gl/oOhltn

Ministerio de Trabajo y Seguridad Social y Ministerio de Salud. (1989). Resolución 1016 de 1989. Por la cual se reglamenta la organización, funcionamiento y forma de los Programas de Salud Ocupacional que deben desarrollar los patronos o empleadores en el pais. Recuperado de goo.gl/iFdHzy

Organización Internacional de Normalización - ISO. (2006). Guía sobre responsabilidad social ISO 26000. Ginebra: ISO.

Puga, C., Peschard, J.y Castro, T. (1999). Hacia la sociología (3.a ed.). México: Longman de México Editores S. A.
Ríos, M. y Sánchez, J. (1997). Eficacia organizacional: Concepto, desarrollo y evaluación. Madrid: Ediciones Díaz de Santos S. A.

Robbins, S. y Judge, T. (2009). Comportamiento organiæacional (13.a ed.). E México: Pearson Prentice Hall.

San Antonio Internacional. (s. f). Equipos de workover. Recuperado de goo.gl/7ehzel

Williams, K. and O'Reilly C. (1998) Demography and diversity in organizations. A review of 40 years of research. Research in Organizational Behavior. Vol 20, pages 77-140.

\section{INFOGRAFÍA}

Agencia Nacional de Hidrocarburos Colombia. Glosario. [En línea]. [Citado el 30 de mayo de 2013]. Disponible en: <http://www.anh.gov.co/es/index. php?id=93>

Banco Wiese Sudameris. Reporte Sectorial del Sector de Hidrocarburos Citado en 31 de mayo 2013. Disponible en <http://www.scotiabank.com.pe/i_ financiera/pdf/sectorial/20020206_sec_es_hidrocarburos.pdf> 